\title{
The unusual supernova remnant G76.9+1.0: The low-frequency radio spectrum
}

\author{
T.L. Landecker ${ }^{1}$, Y. Zheng ${ }^{2}$, X. Zhang ${ }^{2}$, and L.A. Higgs ${ }^{1}$ \\ 1 Dominion Radio Astrophysical Observatory, National Research Council, Box 248, Penticton, British Columbia V2A 6K3, \\ Canada \\ ${ }^{2}$ Beijing Astronomical Observatory, Chinese Academy of Sciences, Beijing 100080, China
}

Received January 19; accepted September 11, 1996

\begin{abstract}
G76.9+1.0 is an unusual radio source which is interpreted as a filled-centre supernova remnant (SNR). New 232-MHz observations of a field containing G76.9+1.0 have been combined with a re-analysis of existing observational data to derive the low-frequency spectrum of the SNR. Its spectral index has a value of about 0.6 above $1 \mathrm{GHz}$, and appears to flatten below that frequency. This may indicate a "break" in the spectrum below $1 \mathrm{GHz}$. However, further observations at frequencies below $400 \mathrm{MHz}$ will be required before this can be firmly established. The low-frequency spectra of several other radio sources in the field surrounding G76.9+1.0 were also derived, partially to confirm the validity of the $232-\mathrm{MHz}$ flux densities but also to provide new lower frequency data for these objects. They include several $\mathrm{H}$ II regions and some extragalactic sources. Two of the latter have rather steep spectra and merit further study.
\end{abstract}

Key words: ISM: supernova remnants - ISM: individual objects: G76.9+1.0 — radio continum: ISM radio continum: general

\section{Introduction}

The interesting object G76.9+1.0 was detected by Wendker et al. (1991) in their survey of continuum emission from the Cygnus $\mathrm{X}$ region. Those observations, with an angular resolution of $3.5^{\prime} \times 5.2^{\prime}$ at $408 \mathrm{MHz}$, established that the source is an extended object, about $5^{\prime}$ to half power, with a non-thermal spectrum (measured between 408 and $4800 \mathrm{MHz}$ ). The structure of the object was revealed in observations of higher angular resolution made by Landecker et al. (1993). These observations showed that G76.9+1.0 is morphologically similar to the sub-class

Send offprint requests to: T.L. Landecker of supernova remnants (SNRs) which resemble the Crab Nebula, the filled-centre remnants.

The emission from G76.9+1.0 has a spectral index $\alpha=0.62 \pm 0.04$ (where $S \propto \nu^{-\alpha}$ ) between 408 and 2700 $\mathrm{MHz}$ (Landecker et al. 1993). However, there is some evidence that the spectral index is lower at lower frequencies: Taylor et al. (1992) measured a flux density at $327 \mathrm{MHz}$ which is approximately the same as the $408-\mathrm{MHz}$ value, and is significantly less than the flux density predicted by extrapolating the high-frequency spectrum. In fact, a recent recalibration of the $327-\mathrm{MHz}$ observations (Taylor et al. 1996) indicates that the value reported by Taylor et al. (1992) should be revised downward. To investigate the spectrum of the source further, we sought to make a flux-density measurement at a lower frequency, and in this paper we report a flux density obtained at $232 \mathrm{MHz}$. To give confidence in our spectrum for G76.9+1.0 we have also obtained flux densities for a number of other sources in the vicinity over as wide a frequency range as possible.

\section{Observations and data reduction}

\subsection{2- $\mathrm{MHz}$ image}

The region around G76.9+1.0 was observed using the Miyun Synthesis Telescope (Zhang et al. 1993). Table 1 gives details of the observations.

Data reduction was complicated by the proximity of Cygnus A to the field centre (at the $30 \%$ level of the primary beam). Phase and amplitude errors in the original data generated a high artefact level in the region of interest, and it was necessary to process the image using the phase and amplitude self-calibration procedures in AIPS. The original data were processed five times, starting with different model inputs. At the conclusion of processing, artefacts in the images due to Cygnus A had been reduced to a background level of $\sim 400 \mathrm{mJy} /$ beam in the immediate vicinity of G76.9+1.0 in each of the images. All $232-\mathrm{MHz}$ flux densities quoted in this paper are the average of measurements made from the five images; the 
Table 1. Details of observations with the Miyun Synthesis Telescope

\begin{tabular}{lll}
\hline Dates of Observation: & & $1993 \mathrm{June} 1,6$ and 7 \\
Field Centre (J2000): & R.A. & $20^{\mathrm{h}} 20^{\mathrm{m}} 15.7^{\mathrm{s}}$ \\
& Dec. & $34^{\circ} 51^{\prime} 57^{\prime \prime}$ \\
& $232 \mathrm{MHz}$ \\
Observing Frequency: & 28 \\
Number of Antennas: & $9 \mathrm{~m}$ \\
Antenna Diameter: & $10^{\circ} \times 12^{\circ}$ at $50 \%$ \\
Field of View: & $3.8^{\prime}(\mathrm{EW}) \times 5.4^{\prime}(\mathrm{NS})$ \\
Synthesized Beam: & 18 to $1164 \mathrm{~m}$ \\
Baseline Coverage: & $6 \mathrm{~m}$ \\
Baseline Increment: & 192 \\
Number of Baselines: & $100 \mathrm{~K}$ \\
Receiver Noise: & $1.5 \mathrm{MHz}$ \\
Bandwidth: & $96-$ baseline, 1-bit \\
Correlator: & digital, with digital \\
& path compensation \\
Sample Time: & $10 \mathrm{~s}$ \\
Visibility Noise: & $10 \mathrm{Jy} /$ baseline/sample \\
Polarization: & linear \\
Calibrator: & Cyg A (7944 Jy) \\
\hline
\end{tabular}

quoted error is the estimate of the standard deviation in one determination of flux density.

The primary telescope calibration was based on a model of Cygnus A, with an integrated flux density based on the scale of Baars et al. (1977). The model is based on many observations with the Miyun Telescope (Zhang et al. 1993). For the present observations, the flux-density scale was further adjusted using three sources in the field (other than G76.9+1.0). Flux densities for these sources are available in the literature at a number of frequencies between 178 and $4850 \mathrm{MHz}$, and a flux density at $232 \mathrm{MHz}$ can be predicted with good accuracy. The measured and predicted flux densities for this set of sources were compared to establish an overall flux-density scale for the 232-MHz image. We refer to these three sources as comparison sources.

Because we were working so close to the sensitivity limit of the telescope at $232 \mathrm{MHz}$, we took several further steps to establish the reliability of our flux densities. Flux densities were measured for a further twelve sources (or source blends in two cases) at $232 \mathrm{MHz}$ and flux densities for these sources were also compiled from the literature, and from re-analysis of images in the same manner as the analysis of the $232-\mathrm{MHz}$ images. Their spectra help establish the reliability of the $232-\mathrm{MHz}$ flux densities.

The source we are studying is extended, while the comparison sources used to establish the flux-density scale are essentially unresolved. It is conceivable that our data processing has had a different effect on resolved and unresolved sources, and we therefore included some extended $\mathrm{H}$ II regions in the list of sources we examined.

Some of the sources included in our list prove to have inherent interest, and we comment on them wherever warranted.
AVERAGE 232-MHz IMAGE
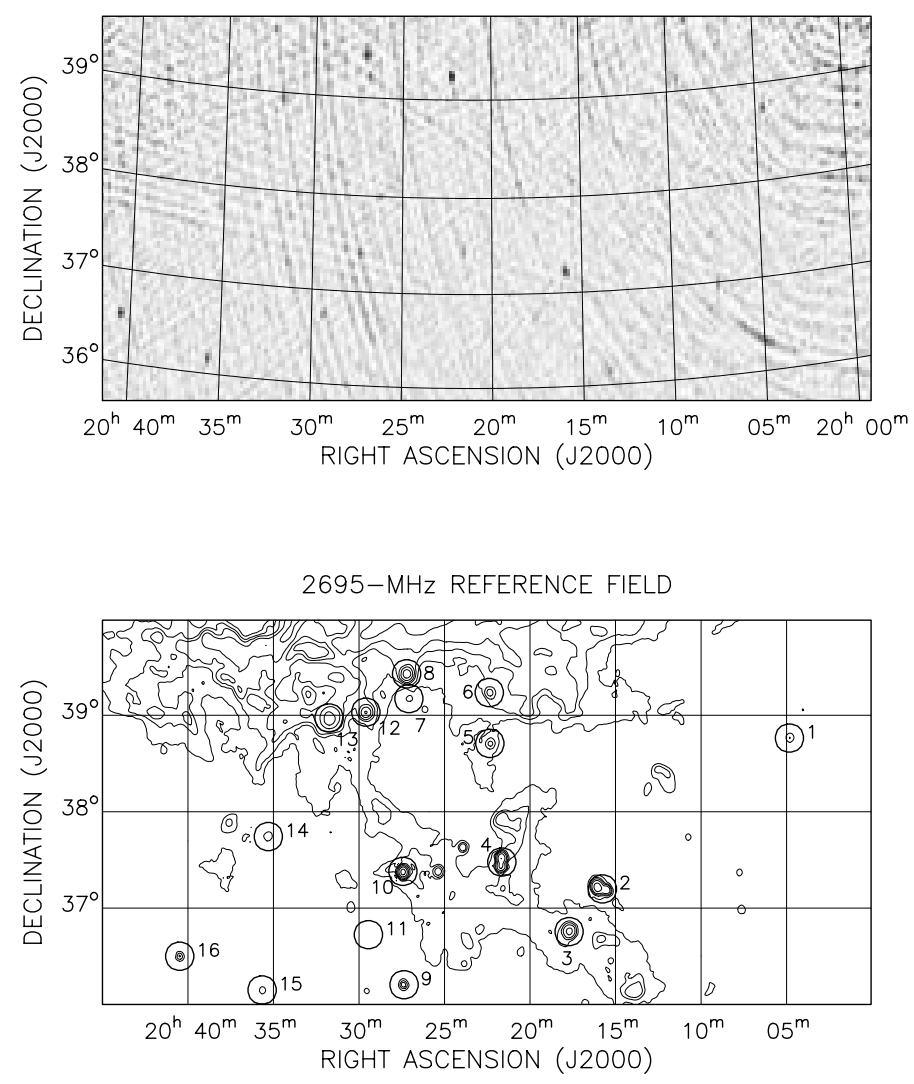

Fig. 1. Top: Average of five 232-MHz images. The gray-scale presentation is from $-0.3 \mathrm{Jy} /$ beam (lightest) to $4 \mathrm{Jy} /$ beam (black). Bottom: Approximately the same area showing contours of $2695-\mathrm{MHz}$ radio emission (Fürst et al. 1990a; Reich et al. 1990b). The contours are at 0.5, 1, 1.5, 2.5, 5, 10, and $20 \mathrm{~K}$ brightness temperature. The sixteen radio sources (or merged sources) that are used in this study are indicated by the numbered circles. The numbers refer to Table 4 . Most of these are visible in the $232-\mathrm{MHz}$ image but some are lost in the noise

\subsection{Source flux densities at other frequencies}

At $408 \mathrm{MHz}$, we used five separate observations made with the DRAO Synthesis Telescope to determine flux densities for the sources of interest. Table 2 gives details of these observations; all have different phase centres.

Table 3 lists all sources of data used in this paper. Where data were available to us in the form of source lists, any subsequent revisions to the flux density scale were applied. Where data were available to us in the form of images, even if published source flux densities existed, the flux density of each source was measured (or re-measured) in two ways. First, a flux density was derived using the DRAO Gaussian-fitting package, which fits a two-dimensional Gaussian function or functions after first removing a smoothly varying background. Second, an integrated flux density was determined using the DRAO polygon integration package, which computes the integrated 
Table 2. $408-\mathrm{MHz}$ observations

\begin{tabular}{lcccc}
\hline Obs. & $\begin{array}{c}\text { Right Asc. } \\
(\mathrm{J} 2000)\end{array}$ & $\begin{array}{c}\text { Declination } \\
(\mathrm{J} 2000)\end{array}$ & Date & Ref. \\
\hline 1 & $20^{\mathrm{h}} 31^{\mathrm{m}} 49^{\mathrm{s}}$ & $40^{\circ} 40^{\prime} 13^{\prime \prime}$ & 1985 July-Aug & 1 \\
2 & $20^{\mathrm{h}} 14^{\mathrm{m}} 11^{\mathrm{s}}$ & $36^{\circ} 12^{\prime} 10^{\prime \prime}$ & 1986 Aug-Oct & 2 \\
3 & $20^{\mathrm{h}} 13^{\mathrm{m}} 55^{\mathrm{s}}$ & $34^{\circ} 49^{\prime} 09^{\prime \prime}$ & 1993 July-Sep & 3 \\
4 & $20^{\mathrm{h}} 27^{\mathrm{m}} 30^{\mathrm{s}}$ & $37^{\circ} 22^{\prime} 23^{\prime \prime}$ & 1994 Apr-May & 3 \\
5 & $20^{\mathrm{h}} 16^{\mathrm{m}} 00^{\mathrm{s}}$ & $37^{\circ} 12^{\prime} 00^{\prime \prime}$ & 1994 May-June & 3 \\
References: & & & \\
1. Wendker et al. (1991) & & \\
2. Pineault \& Chastenay (1990) \\
3. Unpublished data from the DRAO Synthesis Telescope \\
\hline
\end{tabular}

Table 3. Data used to establish source flux densities

\begin{tabular}{|c|c|c|c|}
\hline $\begin{array}{l}\text { Frequency } \\
\quad(\mathrm{MHz})\end{array}$ & $\begin{array}{l}\text { Angular } \\
\text { Resolution } \\
\text { (arcmin) }\end{array}$ & Reference & Notes \\
\hline 178 & $*$ & Gower et al. (1967) & 1 \\
\hline 232 & $2.7 \times 4.5$ & This paper & \\
\hline 327 & $1.0 \times 1.6$ & Taylor et al. (1996) & 2 \\
\hline 365 & $*$ & Douglas et al. (1980) & 3 \\
\hline 408 & $3.4 \times 5.6$ & see Table 2 & \\
\hline 1410 & 9.4 & Reich et al. (1990a) & \\
\hline 2695 & 4.3 & $\begin{array}{l}\text { Fürst et al. (1990a) } \\
\text { Reich et al. (1990b) }\end{array}$ & \\
\hline 4800 & 2.6 & Wendker (1984) & \\
\hline 4850 & $*$ & Gregory \& Condon (1991) & 4 \\
\hline \multicolumn{4}{|c|}{$\begin{array}{l}\text { Notes: } \\
\text { * See original reference } \\
\text { 1. } 4 \text { C catalog, corrected by factor } 1.09 \text { (Roger et al. } 19 \\
\text { 2. A re-calibrated image supplied by A.R. Taylor and } \\
\text { B.J. Wallace } \\
\text { 3. The "Texas" Survey } \\
\text { 4. The GB catalog }\end{array}$} \\
\hline
\end{tabular}

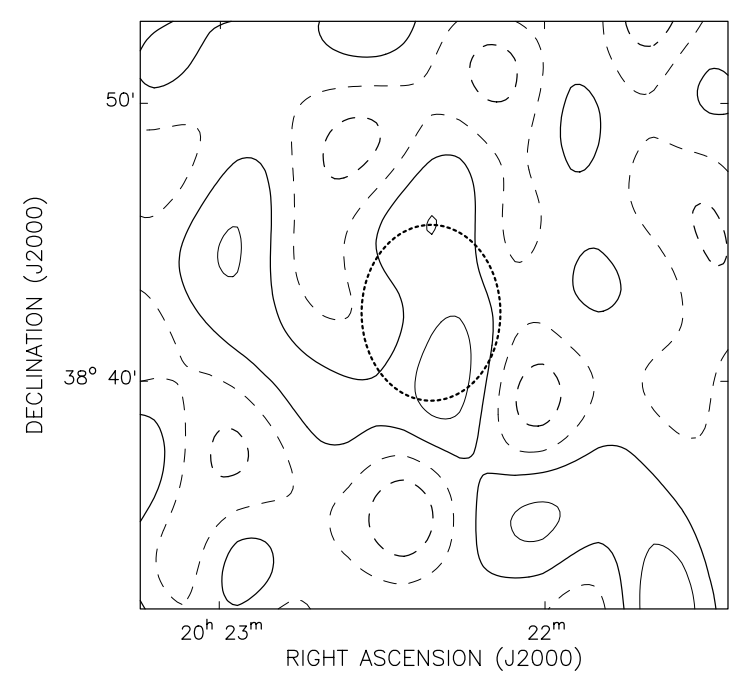

Fig. 2. The new $232-\mathrm{MHz}$ map in the vicinity of the SNR G76.9+1.0, illustrating the dynamic-range problems. The dotted circle denotes the expected position and approximate size of the SNR. The contour levels are $-0.75,-0.25,0.25$ and $0.75 \mathrm{Jy} /$ beam flux density above a smoothly varying background fitted at polygon vertices surrounding a source. The latter procedure was carried out five times, using slightly different polygon vertices, in order to determine an appropriate average value. The adopted flux density for a source was selected from these two results, using weighting judged to be appropriate for each case. In Table 3, if an angular resolution is listed, flux densities were determined from images, otherwise published source lists were used. A systematic $3 \%$ error was added to the statistical errors in flux densities derived from images, and a $4 \%$ possible systematic error was added to the "Texas" flux densities.

\section{Results}

The top portion of Fig. 1 shows our new 232-MHz map of the vicinity of $\mathrm{G} 76.9+1.0$, while the bottom portion shows a similar image at $2695 \mathrm{MHz}$ (Fürst et al. 1990a; Reich et al. 1990b), with the sixteen source positions marked. The $232-\mathrm{MHz}$ image is a weighted mean of the five self-calibrated images. The remaining artefacts in the 232-MHz map due to Cygnus A are very evident.

Figure 2 presents the portion of the $232-\mathrm{MHz}$ map at the position of G76.9+1.0, again illustrating the limited dynamic range of the new observations. The dotted ellipse represents the approximate expected half-power size of the SNR, a circle $4^{\prime}$ in diameter convolved with the telescope response.

Table 4 lists the approximate positions (to an accuracy of about $15^{\prime \prime}$ ), sizes (to an accuracy of about $1^{\prime}$ ), and identifications for all sixteen sources studied in this paper. Two of the sources, Nos. 2 and 4, actually consist of two components at the higher frequencies. The point companion to CTB 87, E 2013+3702 (Morsi \& Reich 1987), is probably unrelated to the SNR. On the other hand, the two bright components of the ON 2 source (No. 4) are probably part of the same HiI complex. Table 5 presents the flux densities used to establish the spectra for the three comparison sources, as well as the $232-\mathrm{MHz}$ flux densities deduced from these spectra. The $232-\mathrm{MHz}$ flux densities for the comparison sources that were derived from

the analysis of the five $232-\mathrm{MHz}$ images were: Source 6 , $8.4 \pm 0.6$; Source 15, $3.9 \pm 0.4$; Source 16, $5.3 \pm 0.7$. Thus flux densities derived directly from the $232-\mathrm{MHz}$ images should be scaled by a factor of $0.83 \pm 0.11$. The three comparison sources have non-thermal spectra, and are probably extragalactic.

Table 6 lists flux densities obtained for the other thirteen sources, including G76.9+1.0. A comparison of flux densities derived in this study with published flux densities, in cases where such lists exist, e.g. the list of Reich et al. (1990a) for the 1410-MHz data and the list of Fürst et al. (1990b) for the 2695-MHz data, indicated no significant differences, except for a few cases where the choice of background could greatly influence derived flux densities. 
There were systematic variations in the derived $408-\mathrm{MHz}$ flux densities from the five DRAO sets of observations. The systematic scale errors that were determined in this study, expressed as the ratio of flux density derived for an individual $408-\mathrm{MHz}$ observation divided by the derived average flux density, are: Obs. 1, $1.10 \pm 0.06$; Obs. 2, $0.95 \pm 0.09$; Obs. 3, $0.83 \pm 0.04$; Obs. 4, $1.07 \pm 0.05$; Obs. $5,1.12 \pm 0.06$. (The observation numbers refer to Table 2 ). These are, of course, based on only a few sources distributed in differing sectors of the individual fields of view, and often at low levels in the primary beam. Therefore they cannot be considered as good estimates of the reliability of DRAO 408-MHz data in general.

The spectra of these thirteen sources, plus those of the calibration sources, are shown in Fig. 3a. In this figure, the dotted lines connect points on a best-fitting quadratic function in the log-log plots, and serve only to highlight spectral trends. They do not correspond to any fitted physical radio-emission spectrum. The spectrum of G76.9+1.0 is included in the figure, and is presented in more detail in Fig. 3b where the weighted best-fit linear spectrum for frequencies greater than $1 \mathrm{GHz}$ is indicated by the solid line. This has a spectral index of $0.64 \pm 0.06$, essentially the same as the value found by Landecker et al. (1993).

It is clear that the $232-\mathrm{MHz}$ flux density lies significantly below the value suggested by the extrapolation of the spectrum at frequencies above $1 \mathrm{GHz}$. The $232-\mathrm{MHz}$ flux density predicted by the higher-frequency fit plotted in Fig. 3b is $4.4 \pm 0.6 \mathrm{Jy}$. The observed value differs from this by over $4 \sigma$. This fact, and the low flux densities at both $327 \mathrm{MHz}$ and $408 \mathrm{MHz}$ suggest a spectrum which is flat or turns over below $\sim 1 \mathrm{GHz}$. This conclusion is, of course, heavily dependent upon the reliability of the re-calibrated $327-\mathrm{MHz}$ data. If the $327-\mathrm{MHz}$ data point is ignored, the best-fitting linear spectrum from $200 \mathrm{MHz}$ to $5 \mathrm{GHz}$ is shown by the dashed line in Fig. 3b. This has a spectral index of $0.48 \pm 0.02$. The $232-\mathrm{MHz}$ value is still a factor of two below this fit (still a deviation of more than $3 \sigma$ ), and the $1.41-\mathrm{GHz}$ point is high. As was noted by Landecker et al. (1993), G76.9+1.0 is superimposed on a ridge of thermal emission which may contribute to deduced flux densities from Gaussian fitting or surface integration, and the "somewhat high" 1.4-GHz flux densities found in that paper were attributed to this. In the present work, all the flux densities have been derived in the same fashion, and one might expect any thermal background contamination to be similar for all the data points above $1 \mathrm{GHz}$, except that the $1.41-\mathrm{GHz}$ beam size is larger than the others.

\section{Discussion}

\subsection{The reliability of the 232-MHz flux density}

From the top portion of Fig. 1 and Fig. 2, it is obvious that the present analysis is severely limited by dynamic-range problems in the vicinity of Cygnus $\mathrm{A}$, and one might ask whether the very low flux density found for G76.9+1.0 (it is barely visible in Fig. 2) is an error of observation. From the plots in Fig. 3a, however, the 232-MHz flux densities appear reliable. Apart from G76.9+1.0, most of the other extended sources are compact $\mathrm{H}$ II regions. They do have very low $232-\mathrm{MHz}$ flux densities but these can generally be attributed to internal self-absorption. The one source for which the $232-\mathrm{MHz}$ flux density seems to be particularly low, however, is Source 13 (DR 13). The H II region spectra are discussed in more detail in Sect. 4.6.3, where it is shown that a low flux density at $232 \mathrm{MHz}$ may be expected for DR 13 because of its environment. Therefore, we believe that the $232-\mathrm{MHz}$ flux densities are generally reliable to the quoted level of error.

On the basis of the data presented in Fig. 3b, one may therefore conclude that the radio spectrum of G76.9+1.0 flattens below $1 \mathrm{GHz}$, or that the $327-\mathrm{MHz}$ flux values are unreliable and the SNR has a spectral index of about 0.5 over the whole frequency range. We consider the former to be more likely.

\subsection{The possibility of foreground absorption}

The Cygnus $\mathrm{X}$ environment is one in which ionized interstellar gas is widespread. Can foreground distributed $\mathrm{H}$ II regions provide significant absorption at $232 \mathrm{MHz}$ ? The background brightness temperature at $408 \mathrm{MHz}$ in the general vicinity of G76.9+1.0 is about $100 \mathrm{~K}$ (Wendker et al. 1991). Assuming an electron temperature of $6000 \mathrm{~K}$ (based on the recombination line measurements of Landecker 1984)) this implies an optical depth $(\tau)$ of 0.017 , which translates to $\tau=0.054$ at $232 \mathrm{MHz}$. Absorption will therefore account for only $\sim 5 \%$ of the apparent flux density deficiency at $232 \mathrm{MHz}$, rather than the $>50 \%$ observed. We conclude that the observed low flux density cannot be attributed to foreground absorption.

\subsection{Is G76.9+1.0 a filled-centre SNR?}

Landecker et al. (1993) discussed the nature of G76.9+1.0, considering that the object could be $(a)$ a filled-centre SNR, (b) a (relatively nearby) normal galaxy, or $(c)$ a large radio galaxy. Interpretation (b) was ruled out because of the very low level of infrared emission, and interpretation (a) was favoured over $(c)$ on morphological grounds, in particular the absence of any discernible edge to the emission, corresponding to the working surface where the jets of a radio galaxy interact with the surrounding medium. On the other hand, the steep radio spectrum above 

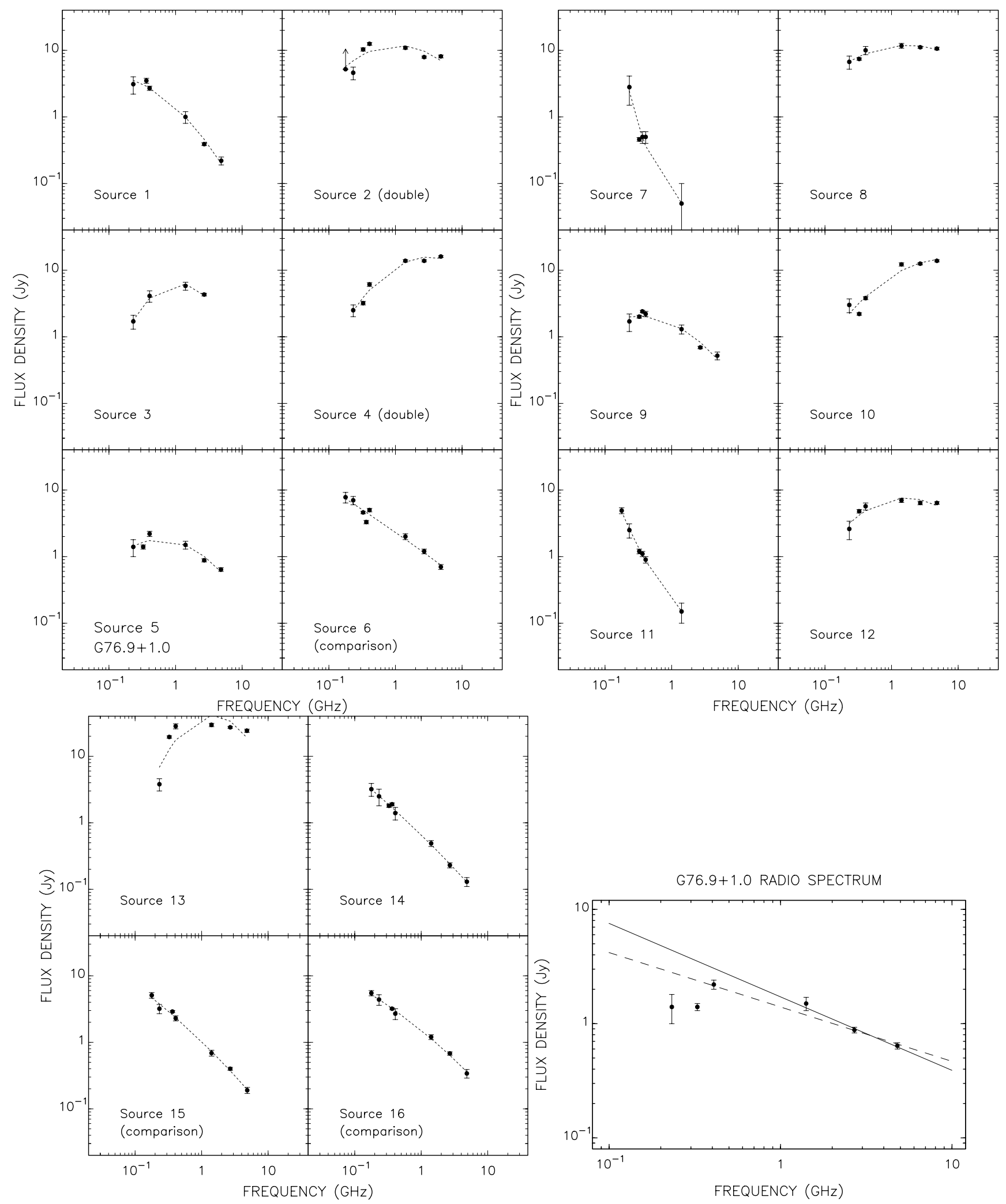

Fig. 3. a) Top and bottom left: Radio spectra of the sixteen radio sources. The dashed lines merely show trends; they connect points on a best-fitting unweighted quadratic curve through the observed data points. For sources with typical thermal spectra that have a sharp transition from optically thin to optically thick, the quadratic curve is a poor representation even of the data trend (cf. Source 13). b) Bottom right: Radio spectrum of the supernova remnant G76.9+1.0. The solid line is a best-fit to the weighted data points above $1 \mathrm{GHz}$. The low-frequency (below $1 \mathrm{GHz}$ ) flux densities fall well below this line and this is may be an intrinsic property of the source. The dashed line gives the spectrum best fitting all the weighted data points, ignoring the $327-\mathrm{MHz}$ point 
Table 4. Approximate positions and sizes of all sources

\begin{tabular}{|c|c|c|c|c|c|}
\hline No. & Positior & (J2000) & Approx. Size & Name(s) & Notes \\
\hline & R.A. & Dec. & (arcmin) & & \\
\hline 1 & 200450 & 384550 & & & \\
\hline $2 \mathrm{~A}$ & 201602 & 371300 & $5.5 \times 4.0$ & 4C 37.57, СТВ 87 & SNR \\
\hline $2 \mathrm{~B}$ & 201530 & 371050 & & E $2013+3702$ & \\
\hline 3 & 201743 & 364520 & $4.8 \times 3.8$ & $\mathrm{G} 74.76+0.62$ & H II region \\
\hline $4 \mathrm{~A}$ & 202140 & 372610 & $1.2 \times 1.1$ & $\mathrm{ON}_{2}$ & H II region \\
\hline $4 \mathrm{~B}$ & 202138 & 373110 & $0.8 \times 0.8$ & ON 2 (north) & H II region \\
\hline 5 & 202221 & 384230 & $4.3 \times 3.3$ & G76.9+1.0 & SNR \\
\hline 6 & 202223 & 391410 & $1.8 \times 1.2$ & $4 \mathrm{C} 39.61$ & comparison source \\
\hline 7 & 202705 & $\begin{array}{lll}39 & 1025\end{array}$ & & & \\
\hline 8 & 202713 & 392550 & $4.0 \times 3.5$ & DR 6 & H II region \\
\hline 9 & 202722 & 361215 & $1.0 \times 0.5$ & & \\
\hline 10 & 202726 & 372250 & $2.3 \times 0.5$ & S 106 & H II region \\
\hline 11 & 202927 & 364325 & & $4 \mathrm{C} 36.40$ & \\
\hline 12 & 202936 & 390200 & $3.5 \times 3.0$ & DR 9 & H II region \\
\hline 13 & 203145 & 385820 & $9.0 \times 8.5$ & DR 13 & H II region \\
\hline 14 & 203518 & 374430 & & $4 C 37.58$ & \\
\hline 15 & 203540 & 360900 & $1.6 \times 1.2$ & 4C 35.49 & comparison source \\
\hline 16 & 204029 & 363000 & $1.5 \times 0.5$ & $4 \mathrm{C} 36.41$ & comparison source \\
\hline
\end{tabular}

Table 5. Flux densities of the comparison sources

\begin{tabular}{|c|c|c|c|c|c|c|c|c|c|}
\hline Source & $178 \mathrm{MHz}$ & $327 \mathrm{MHz}$ & $365 \mathrm{MHz}$ & $408 \mathrm{MHz}$ & $1410 \mathrm{MHz}$ & $2695 \mathrm{MHz}$ & $4800 \mathrm{MHz}$ & $4850 \mathrm{MHz}$ & $\begin{array}{c}\text { Deduced } \\
\text { Flux Density } \\
\text { at } 232 \mathrm{MHz}\end{array}$ \\
\hline 6 & $7.8 \pm 1.4$ & $4.6 \pm 0.2$ & $3.3 \pm 0.2$ & $5.0 \pm 0.3$ & $2.0 \pm 0.2$ & $1.2 \pm 0.1$ & $0.70 \pm 0.06$ & & $5.9 \pm 0.4$ \\
\hline 15 & $5.1 \pm 0.5$ & & $2.9 \pm 0.1$ & $2.3 \pm 0.2$ & $0.69 \pm 0.07$ & $0.40 \pm 0.02$ & & $0.19 \pm 0.02$ & $4.2 \pm 0.3$ \\
\hline 16 & $5.5 \pm 0.5$ & & $3.2 \pm 0.1$ & $2.7 \pm 0.5$ & $1.2 \pm 0.1$ & $0.68 \pm 0.04$ & & $0.34 \pm 0.05$ & $4.5 \pm 0.2$ \\
\hline
\end{tabular}

$400 \mathrm{MHz}$ seemed to favour the radio-galaxy interpretation.

Most SNRs classified as the filled-centre type have flat spectra $(\alpha \leq 0.3)$. This is hardly surprising, since a spectral index in this range is considered a strong indicator of this class of object, as long as it is accompanied by evidence that the emission is non-thermal (usually the detection of polarized emission). However, some filled-centre SNRs show a spectral break (e.g. G74.9+1.2, Morsi \& Reich 1987), with a considerably steeper spectrum above a certain frequency. This fact has become apparent only recently, with the increasing sensitivity of radio telescopes in the millimetre range (Salter et al. 1989). On the basis of the data in Fig. 3b, it is possible that G76.9+1.0 is a member of this class of object, but with a break frequency lower than that of any other known SNR - below $1 \mathrm{GHz}$. It should be noted, however, that, regardless of the precise interpretation of the data in Fig. 3b, G76.9+1.0 has a spectral index above $1 \mathrm{GHz}$ in the range of $0.5-0.6$. This is steeper than normally expected for a filled-centre remnant.

\subsection{Remarks on other sources in the G76.9+1.0 field}

\subsubsection{Source 2: G74.9+1.2 (CTB 87)}

This source is a blend of the filled-centre remnant, G74.9+1.2, and a neighbouring background point source. The latter has a spectrum that peaks near $10 \mathrm{GHz}$ and then decreases towards lower frequencies (with $\alpha \sim-0.3$ ). The spectrum shown in Fig. 3a indicates that the total flux density is dropping at frequencies below $400 \mathrm{MHz}$, whereas the blended spectrum that is expected by extrapolating higher-frequency data (Morsi \& Reich 1987) is still rising at lower frequencies. (Note that the $4 \mathrm{C}$ spectral point is a lower limit because of the "limited" response of that telescope to extended sources). The data presented here suggest that G74.9+1.2 may have a second break frequency in its spectrum. On the other hand, Kovalenko et al. (1994) report relatively high flux densities of $17 \pm 4 \mathrm{Jy}$ and $16 \pm 4 \mathrm{Jy}$ at frequencies of 83 and $111 \mathrm{MHz}$, respectively - consistent with the extrapolated higher-frequency data. The $232-\mathrm{MHz}$ flux density reported in this paper must therefore be considered with caution. 
Table 6. Flux densities of other sources

\begin{tabular}{cccccccccc}
\hline Source & $178 \mathrm{MHz}$ & $232 \mathrm{MHz}$ & $327 \mathrm{MHz}$ & $365 \mathrm{MHz}$ & $408 \mathrm{MHz}$ & $1410 \mathrm{MHz}$ & $2695 \mathrm{MHz}$ & $4800 \mathrm{MHz}$ & $4850 \mathrm{MHz}$ \\
\hline 1 & & $3.1 \pm 0.9$ & & $3.5 \pm 0.3$ & $2.7 \pm 0.2$ & $1.0 \pm 0.2 \dagger$ & $0.39 \pm 0.02$ & \\
2 & $>5.2$ & $4.6 \pm 1.0$ & $10.3 \pm 0.6$ & & $12.5 \pm 0.6$ & $10.9 \pm 0.5$ & $7.9 \pm 0.3$ & $8.1 \pm 0.4$ & $0.22 \pm 0.03$ \\
3 & & $1.7 \pm 0.4$ & & $4.1 \pm 0.8$ & $5.8 \pm 0.8$ & $4.3 \pm 0.2$ & \\
4 & $2.5 \pm 0.5$ & $3.2 \pm 0.2$ & & $6.1 \pm 0.4$ & $13.8 \pm 0.6$ & $13.8 \pm 0.5$ & $16.0 \pm 0.5$ \\
5 & & $1.4 \pm 0.4$ & $1.4 \pm 0.1$ & & $2.2 \pm 0.2$ & $1.5 \pm 0.2$ & $0.88 \pm 0.05$ & $0.64 \pm 0.04$ \\
7 & & $2.8 \pm 1.3$ & $0.46 \pm 0.03$ & $0.5 \pm 0.1$ & $0.5 \pm 0.1$ & $0.05 \pm 0.05$ & & & \\
8 & & $6.7 \pm 1.5$ & $7.4 \pm 0.3$ & & $10.0 \pm 1.4$ & $11.7 \pm 1.0$ & $11.1 \pm 0.4$ & $10.6 \pm 0.5$ \\
9 & & $1.7 \pm 0.5$ & $2.0 \pm 0.1$ & $2.4 \pm 0.1$ & $2.2 \pm 0.2$ & $1.3 \pm 0.2$ & $0.69 \pm 0.03$ & \\
10 & $3.0 \pm 0.7$ & $2.2 \pm 0.1$ & & $3.8 \pm 0.2$ & $12.2 \pm 0.7$ & $12.5 \pm 0.5$ & $13.8 \pm 0.5$ \\
11 & $4.9 \pm 0.5$ & $2.5 \pm 0.6$ & $1.2 \pm 0.1$ & $1.1 \pm 0.1$ & $0.9 \pm 0.1$ & $0.15 \pm 0.05$ & & \\
12 & & $2.6 \pm 0.8$ & $4.8 \pm 0.3$ & & $5.7 \pm 0.7$ & $7.0 \pm 0.5$ & $6.4 \pm 0.4$ & $6.4 \pm 0.3$ \\
13 & & $3.8 \pm 0.8$ & $19.5 \pm 0.8$ & & $28.3 \pm 2.5$ & $29.6 \pm 1.7$ & $27.2 \pm 0.9$ & $24.2 \pm 1.4$ & \\
14 & $3.2 \pm 0.7$ & $2.5 \pm 0.7$ & $1.8 \pm 0.1$ & $1.9 \pm 0.1$ & $1.4 \pm 0.3$ & $0.49 \pm 0.05$ & $0.23 \pm 0.02$ & \\
\hline
\end{tabular}

$\dagger$ From original source list.

\subsubsection{Source 4: ON 2}

The ON 2 region consists of several compact $\mathrm{H}$ II regions, with two main components. Seven components in all have been identified by Matthews \& Spoelstra (1983). The OH maser source, which gives its name to the complex, is near the southern main component. In Fig. 4, the low-frequency radio spectrum of this region is presented, using the results from Table 6, together with data at $610 \mathrm{MHz}$ (Matthews \& Spoelstra 1983) and $10.7 \mathrm{GHz}$ (Matthews et al. 1977). Other existing data from interferometric observations have been omitted since short-spacing flux density is missing. Matthews and Spoelstra gave model parameters for the seven components, and the expected spectrum from those components is given by the dotted curve in Fig. 4 - a poor fit to the data. The fit can be improved, but only by reducing the electron temperature to unreasonably low values. It seems that a model of dense "knots" of emission fits the observed data better. The simplest way of modelling such a clumpy medium is to assume that each of the radio source components consists of a number of small "knots", unresolved in existing observations. The solid curve in the figure shows the spectrum of one such model: 260 spherical clumps, $6^{\prime \prime}$ in diameter, with an electron density of $3000 \mathrm{~cm}^{-3}$ and an electron temperature of $7500 \mathrm{~K}$, at a distance of $5.5 \mathrm{kpc}$. If a nearer distance is assumed, say 900 pc (cf. Dent et al. 1988), the electron density would have to be even higher, $\sim 7400 \mathrm{~cm}^{-3}$. Of course, the combination of number of clumps and clump size is very arbitrary, and other choices would be equally compatible with existing observations.

\subsubsection{Srcs. 3, 8, 12, 13: G74.76+0.62, DR 6, DR 9, DR 13}

These HII regions all have radio spectra showing self-absorption turn-overs. Higher-resolution images of the last three objects exist in the literature: DR 6 (Odenwald et al. 1986), DR 9 and 13 (Wendker et al. 1991). These show that the sources have complex radio structures. If one attempts to fit simple (uniform-density) thermal models to the observed data in Table 6 , one finds, as for the ON 2 complex, that a "clumpy" electron-density distribution is required. If an arbitrary distance of $1.5 \mathrm{kpc}$ is assumed for all of these sources, a model in which the electron density is restricted to spherical clumps $15^{\prime \prime}$ in diameter of uniform electron density, with an electron temperature of $7500 \mathrm{~K}$, gives the solid curves shown in Fig. 5. For the two stronger sources, DR 6 and DR 13, the electron density of the clumps is taken to be $1500 \mathrm{~cm}^{-3}$, while for the other two, $2000 \mathrm{~cm}^{-3}$ was used. The numbers of such clumps required to give the observed flux density were 40 (G74.76+0.62), 160 (DR 6), 60 (DR 9) and 390 (DR 13). If different distances, $D$, should be more appropriate, these electron densities scale as $D^{-0.5}$.

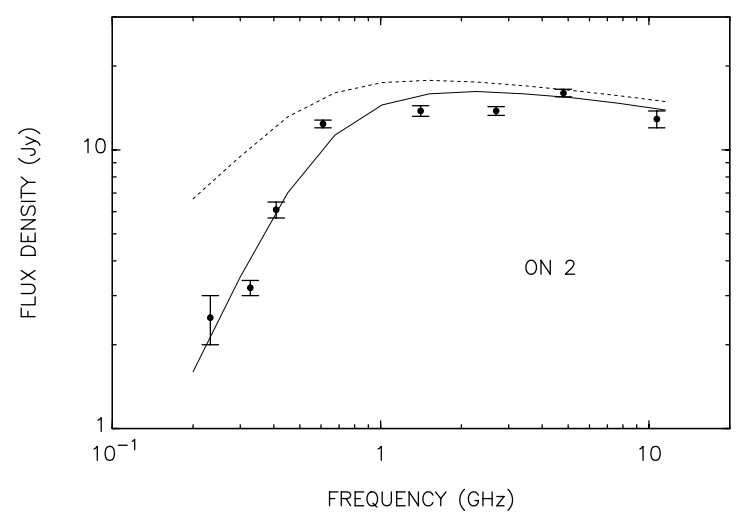

Fig. 4. The low-frequency spectrum of the ON 2 cluster of radio sources. The dotted curve gives the predicted spectrum from the model (of seven components) given by Matthews \& Spoelstra (1983), while the solid curve results from a model consisting of a large number of small, high-density "knots" 
As noted earlier in the paper, the $232-\mathrm{MHz}$ flux density for DR 13 seems extremely low. If a linear spectrum is fitted to the data points in Fig. 5 below $1 \mathrm{GHz}$, a spectral index of $-2.7 \pm 0.4$ is found, steeper than the -2.0 that internal self-absorption allows. The lowest 232$\mathrm{MHz}$ flux density that a thermal spectrum would allow would be close to $10 \mathrm{Jy}$. Indeed, the fitted solid curve in the figure would predict a value close to $15 \mathrm{Jy}$. On the other hand, of these four HII regions, DR 13 is the one which is situated in the strongest area of extended surrounding radio emission. Since flux densities are derived from excess brightness above a surrounding background, the opacity properties of the background must be considered. If, for example, the clumpy model of DR 13 used above were immersed in an extended $\mathrm{H}$ II background of emission measure, $E \sim 510^{4} \mathrm{~cm}^{-6} \mathrm{pc}$ (about one fifth that of the clumps themselves), the clumps will fade at lower frequencies and the dotted spectrum shown in Fig. 5 would result. (This means that the apparent spectrum can drop much faster than the normal black-body curve). Although the $232-\mathrm{MHz}$ point still lies below this modified spectrum, it does indicate that the detailed structure of DR 13 and its environment may cause the source to disappear rapidly as one observes at lower frequencies.

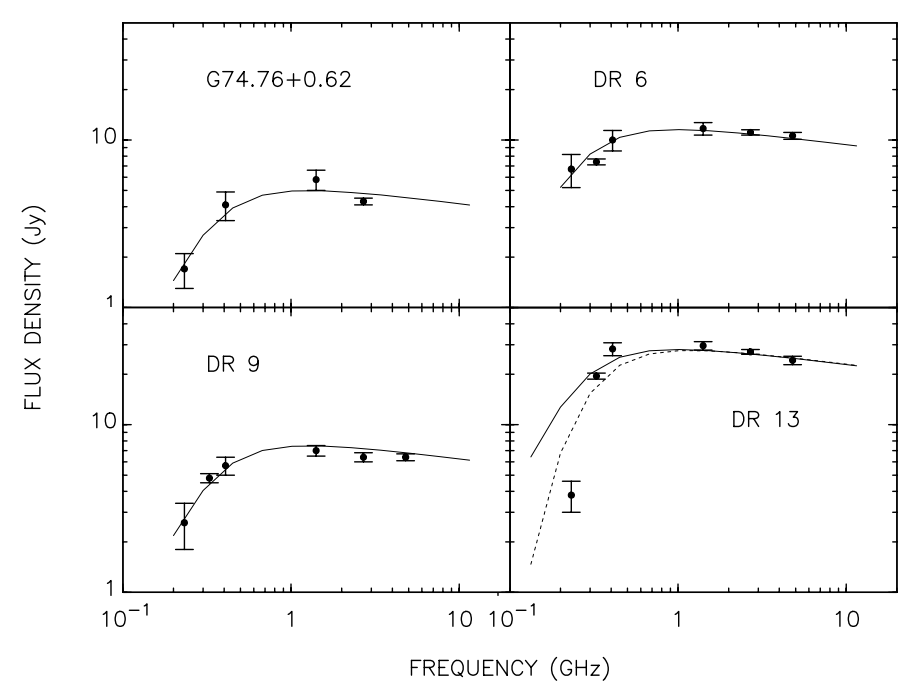

Fig. 5. The low-frequency spectra of the optically-thick H II regions in the area of this study. The solid curves give representative spectra for models consisting of a large number of high-density "knots". For DR 13, the dotted curve shows the effect of immersing these knots or clumps in an extended medium of emission measure of about one fifth of that of the clumps themselves

\subsubsection{Sources 7 and 11: Steep-spectrum sources}

Of the sixteen sources examined in this paper, two exhibit very steep radio spectra, No. 7 and No. 11 (4C 36.40). The spectral indices found for these sources, using the data in Table 6 (without weighting), are $\alpha=1.99 \pm 0.35$ and $\alpha=1.63 \pm 0.11$, respectively. Both appear to be point sources for the resolutions used in this study.

\subsubsection{Source 9: A curved-spectrum source?}

From Fig. 3a, one sees that this source has a non-thermal spectrum above $1 \mathrm{GHz}$, but which appears to flatten at lower frequencies. The source appears to be slightly resolved at the resolutions used in this paper and hence would seem to be a candidate for further investigation possibly a small-diameter SNR. However, Green (1985) has observed this source (G75.43-1.30) at high resolution and has found it to be unresolved. It is therefore likely to be extragalactic.

\subsubsection{Source 10: S 106}

This object is a well-known bipolar nebula (cf. Staude \& Elsässer 1993) and has been the subject of many radio studies (e.g. Israel \& Felli 1978; Bally et al. 1983; Felli et al. 1984). This object consists of two intense radio "lobes" bracketting a weak radio source with a massoutflow spectrum. The lobes have a complex radio structure, with much of the radio emission apparently coming from ionization fronts on the surface of molecular lobes. The data obtained in this paper define the low-frequency spectrum of these radio-continuum lobes. In Fig. 6, a model spectrum is shown along with the observed data points. The model, assumed to be at a distance of $500 \mathrm{pc}$, consists of two conical lobes, with their axes at right angles to the line of sight. The cones have opening angles of $60^{\circ}$, a minimum radius (from the central outflow source) of $5^{\prime \prime}$, a maximum radius of $1.2^{\prime}$, an electron temperature of $10^{5} \mathrm{~K}$, and an electron density varying as $r^{-1.5}$ with a value of $5.510^{4} \mathrm{~cm}^{-3}$ at a radius of $1^{\prime}$. This is, of course, a crude approximation to a very complex object, but gives order-of-magnitude estimates of the physical parameters.

\section{Conclusions}

New observations at $232 \mathrm{MHz}$ have been combined with a re-analysis of several other existing sets of data to obtain the spectrum of the unusual supernova remnant G76.9+1.0 below $5 \mathrm{GHz}$. In order to demonstrate the reliability of the 232-MHz observations and to calibrate them, the spectra of fifteen other radio sources in the area of G76.9+1.0 have also been derived. With one possible exception, the 232-MHz flux densities agree well with those expected from the spectra and characters of these sources. The principal result to emerge from this study is that the spectrum of G76.9+1.0, which is a filled-centre SNR, appears to flatten below $1 \mathrm{GHz}$. Such a spectral break has been found or suspected in other filled-centre remnants. Further low-frequency observations of G76.9+1.0 will be 


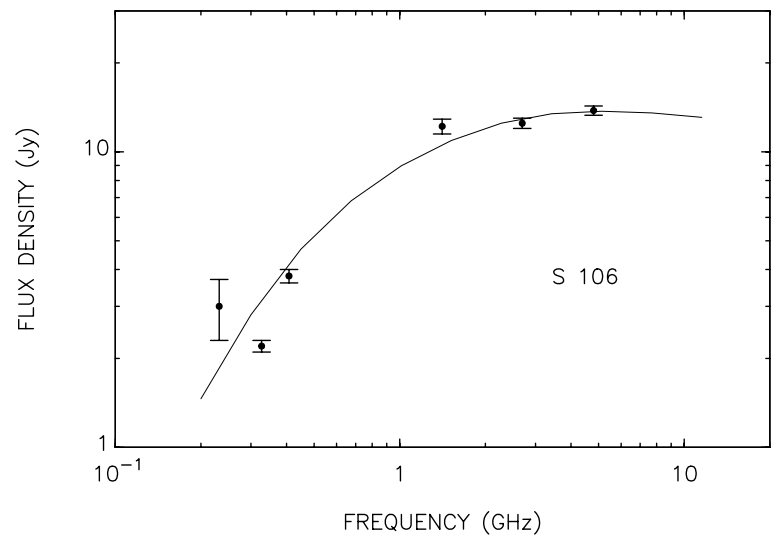

Fig. 6. The low-frequency spectrum of the S $106 \mathrm{H}$ II region. The representative spectrum indicated by the curve is that expected from a bi-conical source with radially-decreasing electron density - a crude approximation to the observed lobe structure

required, however, before such a break can be firmly established.

This study has also contributed new low-frequency information on the integrated spectra of the other sources in the area surrounding G76.9+1.0. These include several H II regions, another filled-centre SNR, and several extragalactic sources. Two steep-spectrum sources probably warrant further investigation. Crude "representative" physical models have been fitted approximately to the $\mathrm{H}$ II region spectra. The most significant conclusion is that very clumpy models are required, consisting of "knots" of emission measure with low filling factors.

Acknowledgements. We are grateful to Dr. S. Pineault for providing unpublished $408-\mathrm{MHz}$ data, and to Drs. A.R. Taylor and B.J. Wallace for providing the $327-\mathrm{MHz}$ data. We thank the referee, E. Fürst, for several suggestions for improvements in the paper. This work was carried out under the China-Canada Scientific Exchange agreement, which operates under the auspices of the Chinese Academy of Sciences and the National Research Council of Canada. Zhang Xi-zhen and Zheng Yijia are grateful to DRAO for hospitality during their stay in Canada. DRAO is operated as a national facility by the National Research Council of Canada.

\section{References}

Baars J.W.M., Genzel R., Pauliny-Toth I.I.K., Witzel A., 1977, A\&A 61,99

Bally J., Snell R.L., Predmore R., 1983, ApJ 272, 154

Dent W.R.F., MacDonald G.H., Andersson M., 1988, MNRAS 235,1397

Douglas J.N., Bash F.N., Torrence G.W., Wolfe C., 1980, Univ. Texas Publ. Astron. No. 17

Felli M., Staude H.J., Reddmann T., et al., 1984, A\&A 135, 261

Fürst E., Reich W., Reich P., Reif K., 1990a, A\&AS 85, 691

Fürst E., Reich W., Reich P., Reif K., 1990b, A\&AS 85, 805

Gower J.F.R., Scott P.F., Wills D., 1967, Mem. R. Astron. Soc. 71,49

Green D.A., 1985, MNRAS 216, 691

Gregory P.C., Condon J.J., 1991, ApJS 75, 1011

Israel F.P., Felli M., 1978, A\&A 63, 325

Kovalenko A.V., Pynzar' A.V., Udal'tsov V.A., 1994, AZh 71, 110

Landecker T.L., 1984, AJ 89, 95

Landecker T.L., Higgs L.A., Wendker H.J., 1993, A\&A 276, 522

Matthews H.E., Spoelstra T.A.T., 1983, A\&A 126, 433

Matthews H.E., Goss W.M., Winnberg A., Habing H.J., 1977, A\&A 61,261

Morsi H.W., Reich W., 1987, A\&AS 69, 533

Odenwald S., Shivanandan K., Campbell M., et al., 1986, ApJ 306,122

Pineault S., Chastenay P., 1990, MNRAS 246, 169

Reich W., Reich P., Fürst E., 1990a, A\&AS 83, 539

Reich W., Fürst E., Reich P., Reif K., 1990b, A\&AS 85, 633

Roger R.S., Bridle A.H., Costain C.H., 1973, AJ 78, 1030

Taylor A.R., Wallace B.J., Goss W.M., 1992, AJ 103, 931

Taylor A.R., Goss W.M., Coleman P.H., van Leeuwen J., Wallace B.J., 1996, ApJS (in press)

Salter C.J., Reynolds S.P., Hogg D.E., Payne J.M., Rhodes P.J., 1989, ApJ 338, 171

Staude, H.J., Elsässer, H., 1993, A\&AR 5, 165

Wendker H.J., 1984, A\&AS 58, 291

Wendker H.J., Higgs L.A., Landecker T.L., 1991, A\&A 241, 551

Zhang X., Zheng Y., Chen H., Wang S., 1993, A\&AS 99, 545 